\title{
Mechanisms of Palmitate-Induced Lipotoxicity in Osteocytes
}

\author{
Ahmed Al Saedi ${ }^{1,2}$, Sandra Bermeo ${ }^{3}$, Lilian Plotkin ${ }^{4}$, \\ Damian E. Myers ${ }^{1,2}$, Gustavo Duque $e^{1,2,5}$ \\ ${ }^{1}$ Australian Institute for Musculoskeletal Science (AIMSS), The University of Melbourne and \\ Western Health, St. Albans, VIC, Australia \\ ${ }^{2}$ Department of Medicine-Western Health, Melbourne Medical School, The University of \\ Melbourne, St. Albans, VIC, Australia \\ ${ }^{3}$ Facultad de Ciencias Básicas y Biomédicas, Universidad Simón Bolívar, Barranquilla, \\ Colombia \\ ${ }^{4}$ Department of Anatomy and Cell Biology, Indiana University School of Medicine in \\ Indianapolis, IN, U.S.A. \\ ${ }^{5}$ Sydney Medical School Nepean, The University of Sydney, Penrith, NSW, Australia
}

Running Title: Mechanisms of Palmitate-Induced lipotoxicity in Osteocytes

Key words: Palmitic acid; osteocytes; lipotoxicity; fatty acids; osteoporosis.

\section{Correspondence to:}

Gustavo Duque, MD, PhD, FRACP, FGSA

Sunshine Hospital, 176 Furlong Road, St Albans, VIC, 3021, Australia

T +61 383958121 | E gustavo.duque@unimelb.edu.au |W http://www.aimss.org.au

This is the author's manuscript of the article published in final edited form as:

Al Saedi, A., Bermeo, S., Plotkin, L., Myers, D. E., \& Duque, G. (2019). Mechanisms of palmitate-induced lipotoxicity in osteocytes. Bone. https://doi.org/10.1016/j.bone.2019.06.016 


\begin{abstract}
Background: Lipotoxicity is defined as cellular toxicity observed in the presence of an abnormal accumulation of fat and adipocyte-derived factors in non-fat tissues. Palmitic acid (PA), an abundant fatty acid in the bone marrow and particularly in osteoporotic bones, affects osteoblastogenesis and osteoblast function, decreasing their survival through induction of apoptosis and dysfunctional autophagy. In this study, we hypothesized that PA also has a lipotoxic effect on osteocytes in vitro.
\end{abstract}

Methods: Initially, we tested the effect of PA on osteocyte-derived factors DKK1, sclerostin and RANKL. Then, we tested whether PA affects survival and causes apoptosis in osteocytes. Subsequently, we investigated the effect of PA on autophagy by detecting the membrane component LC3-II (Western blot) and staining them and lysosomes with Lysotracker Red dye.

Results: PA decreases RANKL, DKK1 and sclerostin expression in osteocytes. In addition, we found that PA induces apoptosis and reduces osteocyte survival. PA also caused autophagy failure identified by a significant increase in LC3-II and a reduced number of autophagosomes/lysosomes in the cytoplasm.

Conclusion: In addition to the effects of PA on RANKL, DKK1 and sclerostin expression, which could have significant deleterious impact on bone cell coupling and bone turnover, PA also induced apoptosis and reduced autophagy in osteocytes. Considering that apoptosis and cell dysfunction are two common changes occurring in the osteocytes of osteoporotic bone, our findings suggest that PA could play a role in the pathogenesis of the disease. Suppression of these effects could bring new potential targets for therapeutic interventions in the future.

Key words: Apoptosis; autophagy; osteocytes; fatty acids; lipotoxicity; palmitic acid. 


\section{1- Introduction}

Bone quality and quantity decreases with aging due to changes in the structural and material properties of bone [1], which is associated with increased skeletal fragility and fractures [2]. With aging, there is an increase in progenitor mesenchymal stem cell (MSC) differentiation to adipocytes over osteoblasts [3]. Increased expansion of adipocyte component in the bone marrow is associated with high levels of fatty acids within the bone marrow milieu, which has a variety of effects on bone cells including a toxic effect on osteoblasts, a phenomenon termed as lipotoxicity [4]. In bone, lipotoxicity involves the inhibition of osteoblast function and survival by adipocyte-secreted factors, predominantly palmitic acid (PA) [5, 6]. In addition, adipocyte-secreted factors can reduce hematopoiesis and increase osteoclastogenesis by various mechanisms, which can alter bone turnover and lead to bone $\operatorname{loss}[4,7]$.

Osteocytes, which derive from osteoblasts, are the most prevalent bone cells and reside within cavities called lacunae [8]. Osteocytes are terminally differentiated from osteoblasts and long cell projections from the osteocytes connect to the bone surface through these processes that travel through canaliculi. Specific surface markers are expressed on osteocytes during the different stages of their differentiation [9]. Osteocytes act as 'sentinels' that can regulate and modulate bone remodeling and also exhibit endocrine functions through the secretion of proteins that regulate bone formation [8]. Like osteoblasts, osteocytes express important cell regulatory molecules including membrane-bound receptor activator of nuclear factor kappa-B ligand (RANKL), Dickkopf WNT signalling pathway inhibitor1 (DKK1) and sclerostin [8]. High levels of apoptotic osteocytes and many compromised empty lacunae are observed in aging and in osteoporotic bone [10,11], suggesting that these processes are associated with changes in the bone microenvironment that affect osteocyte function and survival. Loss of osteocytes in bone is associated with acceleration of bone resorption [12, 
13], with viable osteocytes in the vicinity of apoptotic cells expressing a high RANKL/OPG ratio and releasing osteoclastogenic signals [14]. Additionally, with aging, the reduction in osteoblast numbers affects osteocyte formation leading to a decrease in osteocyte density and mineralization of the osteocyte lacunae [15]

Previously, we and others have reported the intrinsic mechanisms of PA-induced lipotoxicity in osteoblasts, an effect exerted through induction of dysregulated autophagy and apoptosis in human osteoblasts in vitro $[5,6]$. Therefore, given that PA-induced autophagy and apoptosis are also the most common mechanisms of lipotoxicity in other organs and tissues [16, 17], and that autophagy and apoptosis are common features in age-related bone loss and osteoporosis [10], we hypothesized that PA could play an important role in the pathogenesis of osteocyte apoptosis and dysfunctional autophagy observed in aged and osteoporotic bone.

\section{2- Materials and Methods}

2.1 Osteocytes: MLO-Y4-nGFP murine long bone osteocytes (kindly provided by Dr. Linda Bonewald, Indiana University, USA), were plated on $0.01 \%$ collagen-coated surfaces (Sigma, USA) and grown in a-MEM supplemented with 5\% fetal bovine serum (FBS), $5 \%$ bovine calf serum (BCS), penicillin $(100 \mathrm{U} / \mathrm{ml})$ and streptomycin $(100 \mu \mathrm{g} / \mathrm{ml})$. Cells were incubated at $37^{\circ} \mathrm{C}$ under $5 \% \mathrm{CO}_{2}$. In order to maintain stable transfection in MLO-Y4-nGFP, $0.4 \mathrm{mg} / \mathrm{ml}$ G418 antibiotic was added to the media.

2.2 Western blotting: Osteocytes were treated with PA $(250$ and $500 \mu \mathrm{M})$, or vehicle alone, for $48 \mathrm{~h}$. After various treatment conditions, osteocytes were lysed using lysis buffer (1\% protease inhibitors) and centrifuged at $13000 \mathrm{~g}$ for $10 \mathrm{~min}$ to remove insoluble material. Before electrophoresis, protein concentrations were measured using the Pierce ${ }^{\mathrm{TM}} \mathrm{BCA}$ Protein Assay Kit (Thermo Fisher Scientific, Scoresby, VIC, Australia) and samples were 
dissolved in SDS electrophoresis buffer. 10 micrograms of protein per well were separated on SDS-polyacrylamide gels and subsequently electro transferred to a PVDF membrane for 50 min at $300 \mathrm{~mA}$. After blocking with PBS containing 0.1\% Tween 20 and 5\% BSA, membranes were incubated overnight at $4^{\circ} \mathrm{C}$ using primary antibodies against RANKL, AntiSclerostin primary antibody (Santa Cruz, CA, USA, Cat:sc-130258), and anti- DKK1 (Santa Cruz, CA, USA, Cat: sc-374574), or $\beta$-tubulin. The bound antibodies were detected with the appropriate corresponding secondary antibodies $(1: 10,000)$ conjugated with horseradish peroxidase. Blots were developed using enhanced chemiluminescence. For measuring autophagy, the same procedure was followed except a primary antibody anti-LC3 (Santa Cruz, CA, USA) was used instead. Quantification was performed using Image J software. Relative intensities of the bands were determined using tubulin as control.

2.3 Confocal microscopy: Cells were grown on collagen-coated coverslips in the absence or presence of PA (at each of 250 and $500 \mu \mathrm{M}$ ). Cells were then fixed in $4 \%$ paraformaldehyde for 20 min and washed three times with PBS, permeabilized with $0.1 \%$ Triton X-100 in PBS for $10 \mathrm{~min}$, washed and blocked with 5\% BSA for $1 \mathrm{~h}$. Anti-RANKL antibody (Abcam, 1:100, ab45039), was added and left overnight at $4^{\circ} \mathrm{C}$. Alexa555-conjugated anti-mouse antibody (Abcam, 1:1000 ab150110) was used for $45 \mathrm{~min}$ at room temperature as the secondary antibody. RANKL quantification was performed by calculating the fluorescence intensity of RANKL (red channel) using ImageJ software. The red channel was chosen and then the image was threshold (converted to black-and-white) such that the red staining was represented by white pixels, then the histogram function of the software was used to obtain the total count. The same approach was used for the blue channel to count the number of nuclei in the field. Subsequently, the average staining level per cell was calculated. The same threshold setting and analysis was performed for all three different experiments using 6-8 
images per condition.

To determine autophagy, LC3 (Sigma Aldrich, St. Louis, Missouri, USA), was used as the primary antibody for identifying autophagosomes. Alexa 488 conjugated donkey anti-rabbit (Sigma Aldrich, A-21206, St. Louis, Missouri, USA) was used as the secondary antibody. DAPI was added for 3 min, then cells were washed with PBS twice and Golden Pre-Long anti-fade (\# P36930, Invitrogen) was added before analysis using a Leica SP5 confocal microscope. For identification of autophagolysosomes, Lyso-Tracker Red (LTR) dye (Life Technologies) (50 nM in media) was used for $30 \mathrm{~min}$ and cells were washed with PBS twice and antifade was added before analysis using a Leica SP5 confocal microscope. Control samples were incubated with secondary antibody alone. All images are at equal magnification and were processed and acquired identically (60x magnification using immersion oil).

2.4 Effect of PA on osteocyte viability: To determine the effect of PA on osteocyte viability, osteocytes were plated in 96-well plates at a density of $0.5 \times 10^{4}$ cells $/ \mathrm{cm}^{2}$. After an initial $24 \mathrm{~h}$ period in growth media (time 0$)$, cells were treated with either PA $(250$ and $500 \mu \mathrm{M})$ or vehicle alone. Cell viability was evaluated at timed intervals $(24,48 \mathrm{~h})$ by MTS assay (CellTiter 96® AQueous One Solution Cell Proliferation Assay, Promega, Alexandria, NSW, Australia), according to the manufacturer's instructions. Viability was calculated as the percentage change compared with control. This experiment was repeated three times.

2.5 Flow cytometry: Osteocytes were treated with PA $(250$ and $500 \mu \mathrm{M})$ and incubated in the osteocyte media. After $48 \mathrm{~h}$ incubation, apoptotic cells were quantified by Annexin V-PE staining. Briefly, osteocytes were harvested by centrifugation, washed with PBS and stained for 15 min at room temperature with Annexin V-PE and 7-ADD (BD Biosciences, Cat. No. 65875X, USA) in Annexin binding buffer, according to the manufacturer's instructions. The 
samples were then suspended in Annexin binding buffer and the level of fluorescence was determined using a BD FACS Verse flow cytometer with Flow Jo software (BD Biosciences, USA). Annexin V-PE staining intensity from PA treated and untreated cells were compared to show the average fold increase in apoptotic cells. A dual histogram for Annexin V-PE vs 7-AAD was used to compare the apoptosis from PA-treated and untreated cells.

Furthermore, to determine whether autophagy is affected by PA in osteocytes, cells were incubated with LTR dye $(50 \mathrm{nM})$ for 3 min and the level of fluorescence was measured using a BD FACS Verse flow cytometer with Flow Jo software. Briefly, cells were washed in PBS buffer and trypsinized with lysis buffer. The cells were then washed with PBS and suspended in the presence of DNA viability dye, Propidium Iodide (PI) (Life Technologies, Cat. No. BMS500PI, CA, USA). LTR fluorescence intensity values from PA treated and untreated cells were compared to show the average fold increase in autophagy. A dual histogram was acquired to compare lysosomal fluorescence (representing LTR) with DNA viability dye (LTR+/PI+). Flow cytometry acquisition protocols were performed to collect approximately 10000 events.

2.6 TUNEL assay: Cells were grown in collagen-coated plates and treated with PA (250 and $500 \mu \mathrm{M})$ as previously described. Cells were then trypsinized and fixed with $4 \%$ paraformaldehyde for $20 \mathrm{~min}$ at room temperature and then washed with PBS. TUNEL assay (Roche, Basel, Switzerland) for apoptosis was performed according to the manufacturer's instructions. The level of fluorescence was determined using a BD FACS Verse flow cytometer with Flow Jo software (BD Biosciences, USA).

2.7 Statistical analysis: All data are expressed as the mean of three replicate determinations, unless otherwise stated. Statistical analysis was performed by Student's t-test. T-tests were 
performed using a two-tailed distribution with two sample unequal variances. A probability value of $p<0.05$ was considered statistically significant.

\section{3-Results}

3.1 PA reduces the expression of osteocyte-derived factors: Osteocytes are the most important producers of RANKL in bone [18]. Therefore, in this current study we investigated whether PA affects RANKL expression in osteocytes. We found a decrease in RANKL expression in osteocytes incubated with PA (Figure 1A-C). The number of RANKLexpressing cells was significantly reduced by the higher dose of PA (Figure 1A and B). Western blot quantification showed a significant decrease in RANKL protein expression when the cells were incubated with 250 and $500 \mu \mathrm{M}$ of PA $(p<0.05)$ (Figure 1C and D). In addition, there was a significant decrease in DKK1 and sclerostin protein expression when cells were incubated with two concentrations of PA $(250$ and $500 \mu \mathrm{M})(p<0.05)$ (Figure $1 \mathrm{E}$ and F)

3.2 PA reduces survival and induces apoptosis in osteocytes: Initially, we determined cell viability in osteocytes treated with PA for 24 and $48 \mathrm{~h}$ (Figure 2A). Treatment with $250 \mu \mathrm{M}$ of PA resulted in $10 \%$ reduction of viability at $48 \mathrm{~h}$ as compared to control. In addition, treatment with $500 \mu \mathrm{M}$ of PA resulted in $13 \%$ and $22 \%$ reduction of viability at 24 and $48 \mathrm{~h}$, respectively, as compared to control $(p<0.01)$.

Apoptosis was quantified using two separate techniques: Annexin and TUNEL assays. Osteocytes were exposed to two different concentrations of PA (250 and $500 \mu \mathrm{M})$ or vehicle for $48 \mathrm{~h}$. Figure 2 (B and C) shows Annexin V positive (apoptotic cells) quantified using flow cytometry. The total number of apoptotic cells comprises those in early and late apoptosis (Figure 2C). While control cells showed $13.8 \%$ apoptosis (early $5.6 \%$ and late $8.2 \%$ ), cells 
cultured with $250 \mu \mathrm{M}$ PA showed $21.9 \%$ apoptosis (early $6.7 \%$ and late $15.2 \%, p<0.01$ ), and cells cultured with $500 \mu \mathrm{M}$ PA showed $25 \%$ apoptosis (early $8 \%$ and late $17 \%, p<0.01$ ). Quantification for apoptosis using TUNEL at $48 \mathrm{~h}$ (Figure 2D and E) found 10\% for the vehicle-treated cells vs. $24 \%$ and $36 \%$ for cells incubated with $250 \mu \mathrm{M}$ and $500 \mu \mathrm{M}$ PA respectively $(p<0.01)$.

\subsection{PA decreases lysosome loading and recruitment in osteocytes: Osteocytes were} incubated in the presence or absence of PA $(250$ and $500 \mu \mathrm{M})$ for $24 \mathrm{~h}$ and $48 \mathrm{~h}$ and analyzed by flow cytometry for expression of lysosomal loading by using a lysotracker dye. Flow cytometry analysis showed that PA caused significantly higher levels of lysosome loading compared to control cells (Figure 3 A and B) $(p<0.01)$.

3.4 PA inhibits autophagy in osteocytes: We then investigated whether the increasing levels of lysosome loading in the cytoplasm induced by PA treatment were followed by effective autophagy. In this case, osteocytes were cultured with three concentrations of PA $(100,250$ and $500 \mu \mathrm{M})$. A lower dose of PA was used in these experiments to determine cell sensitivity to autophagy under a dose of PA that has not been toxic in previous studies[5, 19]. Initially, expression of LC3 protein levels was measured as an indicator of autophagosomes in osteocytes. Western blotting quantification showed an increased expression of LC3-II protein when exposed to higher concentrations of PA but with no difference between the lower concentration of PA and the vehicle-treated controls (Figure 4 A and B). These findings were confirmed as indicated by higher levels of LC3-II protein expression (punctate fluorescence staining using specific antibody) in the cytoplasm of PA-treated cells (Figure 4C). To determine whether formation of autophagosomes and lysosomes recruitment was concomitant with efficient formation of autophagolysosomes, cells were incubated in the 
presence or absence of PA for $48 \mathrm{~h}$ with a lysotracker dye LTR and in combination with LC3 staining. Merged images for PA-treated cells showed lower colocalization of LC3-II and the lysotracker probe compared to osteocytes incubated with vehicle (control) (Figure 4C and D).

\section{4- Discussion}

In this study we investigated the lipotoxic effect of PA on osteocytes. Since we have already identified apoptosis and dysfunctional autophagy as the two main mechanisms involved in PA-induced lipotoxicity in osteoblasts in vitro [6], in this study we investigated whether the same mechanisms are involved in osteocyte lipotoxicity. Our results indicate that, as in osteoblasts, PA induces apoptosis and inhibits autophagy in osteocytes in vitro, which could partially explain the high levels of apoptosis and autophagy observed in osteocytes of aged and osteoporotic bone.

MLO-Y4 cells were utilized in this study as these cells model the properties of osteocytes and have proven to be useful for studying apoptosis and cell function related to mature osteocytes $[12,20,21]$. The presence of PA in the media affected the capacity of the osteocytes to express proteins that are essential for the regulation of bone turnover. Lower levels of RANKL could determine a low bone resorption and potential uncoupling. Furthermore, considering that DKK1 and sclerostin play crucial roles in bone remodeling, bone formation, and bone growth through their effects on osteoblast activity and survival [22, 23], lower levels of these proteins induced by the presence of PA could significantly affect bone turnover. However, the implications of these findings on bone turnover could only be determined by future in vivo studies.

Apoptosis is an essential mechanism for bone health [24], however, increased osteocyte apoptosis could have a deleterious effect on bone as observed in aging mice where osteocyte apoptosis was accelerated by loss of sex steroids [25]. In addition, osteocyte apoptosis was 
observed in high-dose corticosteroid treatment in human biopsies and mice samples [26].

Although osteocyte apoptosis could be induced by several factors, such as cytokines, hormones, pharmacological agents, and structural changes of bone [27], the mechanisms of age-related osteocyte apoptosis remain partially explored thus allowing us to hypothesize that this phenomenon could be associated with fatty acid-induced lipotoxicity, which could be the consequence of a fatty (and thus lipotoxic) bone marrow milieu. To test whether osteocytes are sensitive to toxic fatty acids, we used a similar approach to our previous work using PA, which is the most prevalent fatty acid within the human bone marrow milieu [28, 29]. Our dosing was calculated as equivalent to the concentrations of PA in the human bone marrow[29], with a previously demonstrated lipotoxic effect on osteoblasts in vitro [5, 6, 19]. Overall, our results indicate that PA induces apoptosis in osteocytes in vitro, which could explain their lower rates of survival in the presence of PA in the media.

PA also induces lipotoxicity on osteoblasts via dysfunctional autophagy [6]. Autophagy is a lysosomal degradation process necessary for recycling cellular products. Autophagy is generally considered as a survival mechanism, which protects from apoptosis by removing damaged organelles, although in extreme stress it leads to cell death [30]. During the autophagy process, toxic cytoplasmic contents and damaged organelles trigger lysosomes recruitment, which then bind with phagosomes to form single membrane autophagosomes. The autophagic process requires many lysosomes to fuse with autophagosomes. It has been hypothesized that stimulation of autophagy would be anti-aging whereas a reduction in autophagy would promote the aging process[31,32]. Indeed, healthy levels of autophagy are required to maintain an appropriate bone metabolism [33], however, as observed in agerelated bone loss and osteoporosis, compromised autophagy could significantly affect bone turnover and bone mass[11, 34]. PA-exposed osteocytes are expected to suffer from cellular stress. In an early stage, their 
response to PA could consist of lysosomes recruitment while attempting to remove the PA from the cytoplasm and protect the cells from lipotoxicity and cell death. If this mechanism of cell protection is affected by PA, it may eventually lead to cell death, as we have previously observed in human osteoblasts in vitro [6]. In our study, we identified that exposure of osteocytes to PA induced significantly higher levels of recruitment of lysosomes in the cytoplasm but without an effective formation of autophagolysosomes, thus suggesting that the presence of PA affected the autophagy process. These alterations in autophagy induced by PA treatment could then explain in part the higher level of apoptosis observed in the PA-treated cells.

In summary, we have found that, as in osteoblasts, PA induces lipotoxicity in osteocytes. Exposure of osteocytes to PA decreased their expression of osteocyte-derived factors, which are critical to normal bone turnover. In addition, PA induced lipotoxicity through apoptosis and inhibition of autophagy clearly mimicking the changes observed in osteocytes of aging and osteoporotic bone. However, the significance of these findings could only be determined using in vivo models. Most importantly, identification of a potential infiltration of marrow toxic fatty acids within the fluid flow through lacunae and canaliculi of bone, thus reaching the osteocytes and affecting their function and survival, will be a crucial component of future in vivo studies.

In conclusion, PA induces similar lipotoxic effects on osteoblasts and osteocytes in vitro. Although demonstration of this lipotoxic effect in vivo is still lacking, our results allow us to suggest that suppression of this lipotoxic effect in vivo could provide the basis to novel therapeutic approaches to osteoporosis in the future. 


\section{5- Acknowledgements}

This study was supported by the Australian National Health and Medical Research Council (NHMRC 632767), the Nepean Medical Research Foundation, and the Australian Institute for Musculoskeletal Science (AIMSS). 


\section{6- References}

[1] E.A. Zimmermann, E. Schaible, H. Bale, H.D. Barth, S.Y. Tang, P. Reichert, B. Busse, T. Alliston, J.W. Ager, 3rd, R.O. Ritchie, Age-related changes in the plasticity and toughness of human cortical bone at multiple length scales, Proceedings of the National Academy of Sciences of the United States of America 108(35) (2011) 14416-21.

[2] D.P. Fyhrie, B.A. Christiansen, Bone Material Properties and Skeletal Fragility, Calcified tissue international 97(3) (2015) 213-28.

[3] S. Muruganandan, R. Govindarajan, C.J. Sinal. Bone Marrow Adipose Tissue and Skeletal Health. Current Osteoporosis Reports. 16(4) (2018) 434-442.

[4] L. Singh, S. Tyagi, D. Myers, G. Duque. Good, Bad, or Ugly: the Biological Roles of Bone Marrow Fat. Current Osteoporosis Reports. 16(2) (2018) 130-137.

[5] K. Gunaratnam, C. Vidal, J.M. Gimble, G. Duque, Mechanisms of palmitate-induced lipotoxicity in human osteoblasts, Endocrinology 155(1) (2014) 108-16.

[6] K. Gunaratnam, C. Vidal, R. Boadle, C. Thekkedam, G. Duque, Mechanisms of palmitate-induced cell death in human osteoblasts, Biology open 2(12) (2013) 1382-9.

[7] A.G. Veldhuis-Vlug, C.J. Rosen, Clinical implications of bone marrow adiposity, Journal of internal medicine 283(2) (2018) 121-139.

[8] L.F. Bonewald, The amazing osteocyte, Journal of bone and mineral research : the official journal of the American Society for Bone and Mineral Research 26(2) (2011) 229-38.

[9] L.F. Bonewald, Osteocytes as dynamic multifunctional cells, Annals of the New York Academy of Sciences 1116 (2007) 281-90.

[10] R.S. Weinstein, S.C. Manolagas, Apoptosis and osteoporosis, The American journal of medicine 108(2) (2000) 153-64.

[11] S.C. Manolagas, A.M. Parfitt, What old means to bone, Trends in endocrinology and metabolism: TEM 21(6) (2010) 369-74.

[12] L.I. Plotkin, Apoptotic osteocytes and the control of targeted bone resorption, Current osteoporosis reports 12(1) (2014) 121-6.

[13] G. Gu, M. Mulari, Z. Peng, T.A. Hentunen, H.K. Vaananen, Death of osteocytes turns off the inhibition of osteoclasts and triggers local bone resorption, Biochemical and biophysical research communications 335(4) (2005) 1095-101.

[14] O.D. Kennedy, M.B. Schaffler, The roles of osteocyte signaling in bone, The Journal of the American Academy of Orthopaedic Surgeons 20(10) (2012) 670-1. 
[15] H.M. Frost, Tetracycline-based histological analysis of bone remodeling, Calcified tissue research 3(3) (1969) 211-37.

[16] R.H. Unger, L. Orci, Lipoapoptosis: its mechanism and its diseases, Biochimica et biophysica acta 1585(2-3) (2002) 202-12.

[17] L. Martino, M. Masini, M. Novelli, P. Beffy, M. Bugliani, L. Marselli, P. Masiello, P. Marchetti, V. De Tata, Palmitate activates autophagy in INS-1E beta-cells and in isolated rat and human pancreatic islets, PloS one 7(5) (2012) e36188.

[18] T. Nakashima, M. Hayashi, T. Fukunaga, K. Kurata, M. Oh-Hora, J.Q. Feng, L.F. Bonewald, T. Kodama, A. Wutz, E.F. Wagner, J.M. Penninger, H. Takayanagi, Evidence for osteocyte regulation of bone homeostasis through RANKL expression, Nature medicine 17(10) (2011) 1231-4.

[19] A. Elbaz, X. Wu, D. Rivas, J.M. Gimble, G. Duque, Inhibition of fatty acid biosynthesis prevents adipocyte lipotoxicity on human osteoblasts in vitro, Journal of cellular and molecular medicine 14(4) (2010) 982-91.

[20] H.M. Davis, R. Pacheco-Costa, E.G. Atkinson, L.R. Brun, A.R. Gortazar, J. Harris, M. Hiasa, S.A. Bolarinwa, T. Yoneda, M. Ivan, A. Bruzzaniti, T. Bellido, L.I. Plotkin, Disruption of the Cx43/miR21 pathway leads to osteocyte apoptosis and increased osteoclastogenesis with aging, Aging cell 16(3) (2017) 551-563.

[21] L.F. Bonewald, Establishment and characterization of an osteocyte-like cell line, MLOY4, Journal of bone and mineral metabolism 17(1) (1999) 61-5.

[22] M.K. Sutherland, J.C. Geoghegan, C. Yu, E. Turcott, J.E. Skonier, D.G. Winkler, J.A. Latham, Sclerostin promotes the apoptosis of human osteoblastic cells: a novel regulation of bone formation, Bone 35(4) (2004) 828-35.

[23] M.M. McDonald, A. Morse, A. Schindeler, K. Mikulec, L. Peacock, T. Cheng, J. Bobyn, L. Lee, P.A. Baldock, P.I. Croucher, P.P.L. Tam, D.G. Little, Homozygous Dkk1 Knockout Mice Exhibit High Bone Mass Phenotype Due to Increased Bone Formation, Calcified tissue international 102(1) (2018) 105-116.

[24] L.I. Plotkin, R.S. Weinstein, A.M. Parfitt, P.K. Roberson, S.C. Manolagas, T. Bellido, Prevention of osteocyte and osteoblast apoptosis by bisphosphonates and calcitonin, The Journal of clinical investigation 104(10) (1999) 1363-74.

[25] M. Almeida, L. Han, M. Martin-Millan, L.I. Plotkin, S.A. Stewart, P.K. Roberson, S. Kousteni, C.A. O'Brien, T. Bellido, A.M. Parfitt, R.S. Weinstein, R.L. Jilka, S.C. Manolagas, Skeletal involution by age-associated oxidative stress and its acceleration by loss of sex steroids, The Journal of biological chemistry 282(37) (2007) 27285-97. 
[26] R.S. Weinstein, R.L. Jilka, A.M. Parfitt, S.C. Manolagas, Inhibition of osteoblastogenesis and promotion of apoptosis of osteoblasts and osteocytes by glucocorticoids. Potential mechanisms of their deleterious effects on bone, The Journal of clinical investigation 102(2) (1998) 274-82.

[27] O. Verborgt, G.J. Gibson, M.B. Schaffler, Loss of osteocyte integrity in association with microdamage and bone remodeling after fatigue in vivo, Journal of bone and mineral research : the official journal of the American Society for Bone and Mineral Research 15(1) (2000) 60-7.

[28] R. Deshimaru, K. Ishitani, K. Makita, F. Horiguchi, S. Nozawa, Analysis of fatty acid composition in human bone marrow aspirates, The Keio journal of medicine 54(3) (2005) $150-5$.

[29] J.F. Griffith, D.K. Yeung, A.T. Ahuja, C.W. Choy, W.Y. Mei, S.S. Lam, T.P. Lam, Z.Y. Chen, P.C. Leung, A study of bone marrow and subcutaneous fatty acid composition in subjects of varying bone mineral density, Bone 44(6) (2009) 1092-6.

[30] Y. Tsujimoto, S. Shimizu, Another way to die: autophagic programmed cell death, Cell death and differentiation 12 Suppl 2 (2005) 1528-34.

[31] Y.S. Rajawat, Z. Hilioti, I. Bossis, Aging: central role for autophagy and the lysosomal degradative system, Ageing research reviews 8(3) (2009) 199-213.

[32] F. Madeo, N. Tavernarakis, G. Kroemer, Can autophagy promote longevity?, Nature cell biology 12(9) (2010) 842-6.

[33] V. Pierrefite-Carle, S. Santucci-Darmanin, V. Breuil, O. Camuzard, G.F. Carle, Autophagy in bone: Self-eating to stay in balance, Ageing research reviews 24(Pt B) (2015) 206-17.

[34] M. Onal, M. Piemontese, J. Xiong, Y. Wang, L. Han, S. Ye, M. Komatsu, M. Selig, R.S. Weinstein, H. Zhao, R.L. Jilka, M. Almeida, S.C. Manolagas, C.A. O'Brien, Suppression of autophagy in osteocytes mimics skeletal aging, The Journal of biological chemistry 288(24) (2013) 17432-40. 


\section{Figure legends}

Figure 1. PA reduces the expression of osteocyte-derived factors. (A) Osteocytes MLO-Y4nGFP cells were treated with different concentrations of PA (250 and $500 \mu \mathrm{M})$ or control. Cells were fixed with 4\% paraformaldehyde and stained with anti-RANKL. (B) Quantification of RANKL+ cells (\%) from three different experiments using Image J software $(* p<0.05$ vs control). (C) Osteocytes MLO-Y4-nGFP cells were treated with two different concentrations of PA $(250$ and $500 \mu \mathrm{M})$ or control. The figure illustrates the detection of RANKL by Western blot. Tubulin was used as a loading control. (D) Quantification of three different Western blot experiments using Image $\mathbf{J}$ software to analyze Western blot bands $\left({ }^{*} p<0.05\right.$ vs control). (E) Osteocytes MLO-Y4-nGFP cells were treated with two different concentrations of PA $(250$ and $500 \mu \mathrm{M})$ or control, and Western blots were performed against Sclerostin and DKK1. Tubulin was used as a loading control. (F) Quantification of three different experiments using Image $\mathrm{J}$ software $\left({ }^{*} p<0.05\right.$ vs control).

Figure 2. PA reduces survival and induces apoptosis in osteocytes. (A) MLO-Y4 osteocytes were treated with PA for $24 \mathrm{~h}$ and $48 \mathrm{~h}$ and MTS assays were performed. Cell survival was significantly reduced under PA-treated conditions. Quantification of three different experiments $\left({ }^{*} p<0.05\right.$ vs control; $\sigma \mathrm{p}<0.05,250 \mu \mathrm{M}$ vs. $\left.500 \mu \mathrm{M}\right)$. (B and C) Flow cytometry analysis of apoptosis using a two-color (dual color fluorescence) histogram where the horizontal axis represents Annexin V-PE and the vertical axis represents 7-AAD. (B) Histograms shown are unlabeled cells, control cells, cells treated with $250 \mu \mathrm{M}$ PA, and cells treated with $500 \mu \mathrm{M}$ PA. (C) PA treatment induced a significantly higher number of Annexin $\mathrm{V}+$ cells as compared with vehicle-treated cells $\left({ }^{*} p<0.01 \mathrm{vs}\right.$ control).

These results were confirmed by quantification of percentage of apoptotic cells by TUNEL assay (D, E) Osteocytes were treated with different concentrations of PA (250 and $500 \mu \mathrm{M})$ 
or vehicle for $48 \mathrm{~h}$. Cells were fixed in $4 \%$ paraformaldehyde, permeabilized with Triton X100, and a TUNEL assay was performed (D) and percentage of TUNEL positive cells was quantified by flow cytometry (E). Data shown are mean \pm SD from three separate experiments. $(* p<0.01$ vs. control).

Figure 3. PA treatment decreases lysosome content in osteocytes. (A) Cell were treated with PA for 48 hours and analyzed by FACS analysis to measure excitation profiles for lyso-track red (LTR) dye. (B) PA treatment significantly increased lysosome content in osteocytes as compared with vehicle-treated cells. Data shown are mean \pm SD from three separate experiments. $\left({ }^{*} p<0.01\right.$ vs. control).

Figure 4. Effect of PA on autophagy in osteocytes. (A) Osteocytes (MLO-Y4 cells) were treated with two different concentrations of PA $(250$ and $500 \mu \mathrm{M})$ or vehicle (control) and Western blot was performed against LC3 at 24, 48 and 72 Hrs. Tubulin was used as a loading control. (B) Quantification of three different Western blot experiments using Image J software. Treatment with PA increased the expression of the autophagy protein LC3II in a concentration-dependent manner $(* p<0.05$ vs control). (C) Images of osteocytes treated with two different concentrations of PA $(250$ and $500 \mu \mathrm{M})$ or control. Osteocytes were fixed with 4\% paraformaldehyde and stained with anti-LC3, LTR and DAPI. All images are at equal magnification and were processed and acquired identically (60x oil) using a Leica confocal microscope and merged images were obtained using Image J software. (D) Quantification of three different experiments with LC3 expression using Image J software showing lower colocalization of LC3 and LTR indicative of dysfunctional autophagy ( $* \mathrm{p}<0.01$ vs control). 


\section{Highlights}

- Lipotoxicity is defined as cellular toxicity observed in the presence adipocyte-derived factors in non-fat tissues.

- Fatty acids affect osteocyte function and survival

- Suppression of lipotoxicity could bring new potential targets for therapeutic interventions for osteoporosis in the future. 
A

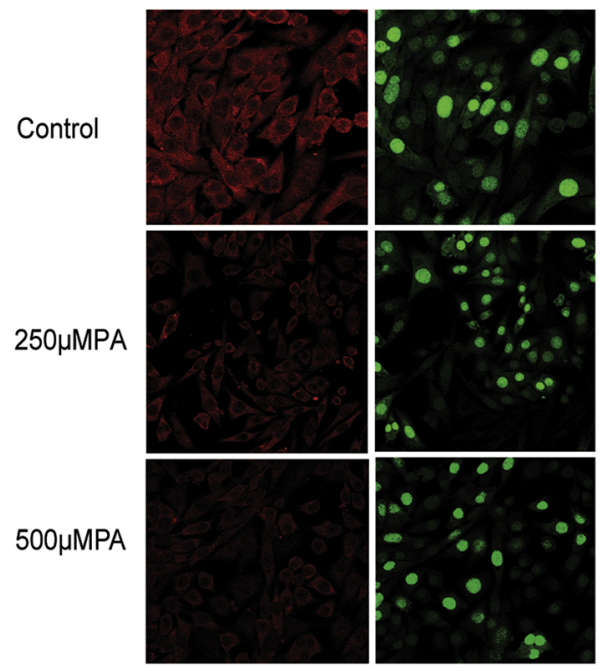

D

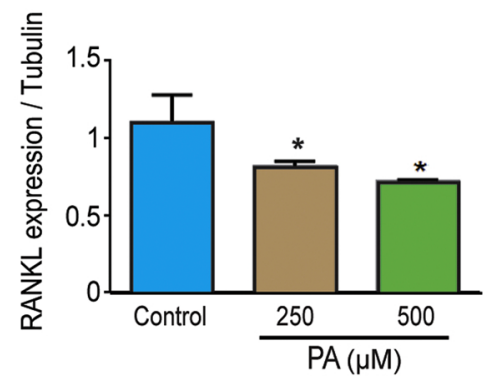

$E$

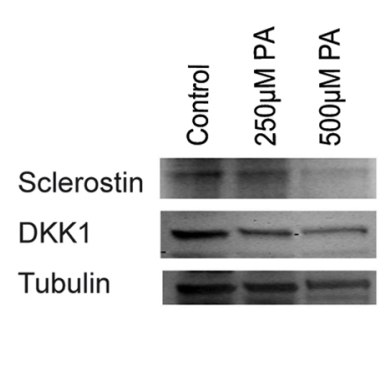

B

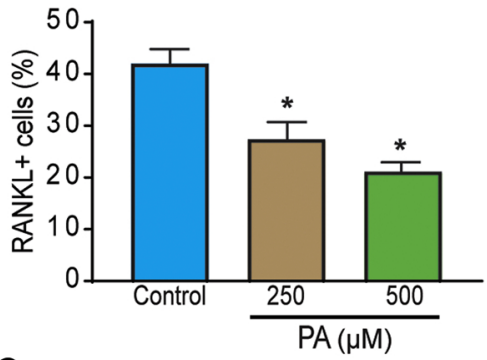

흘 产 产

RANKL

Tubulin

F

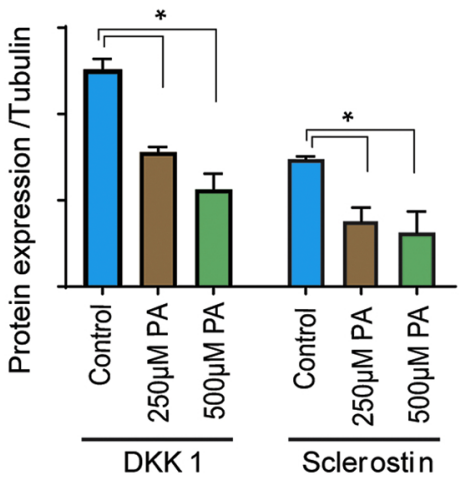

Figure 1 

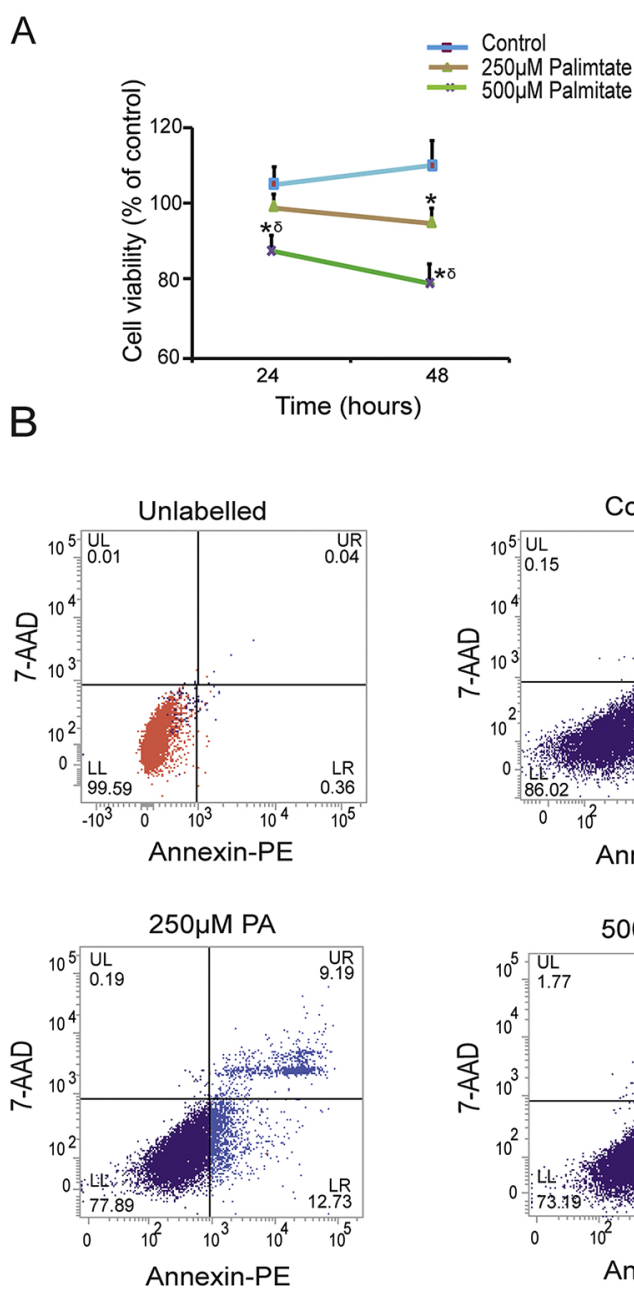

C

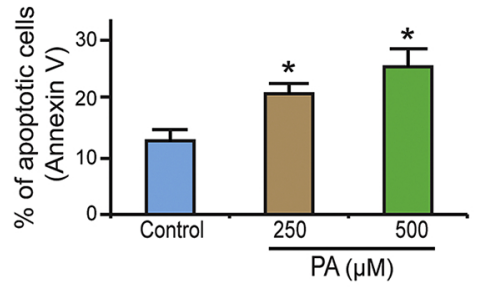

D

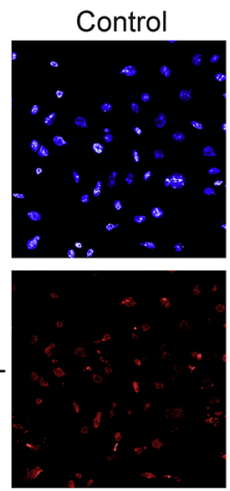

250uM PA

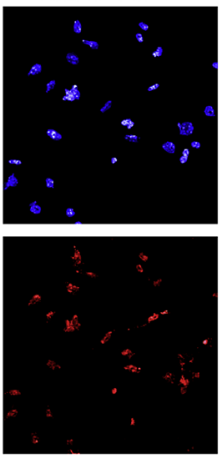

500uM PA

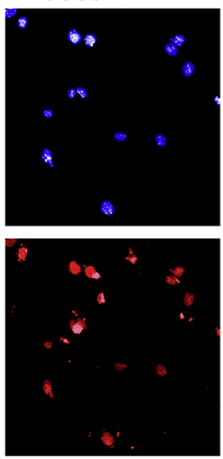

E

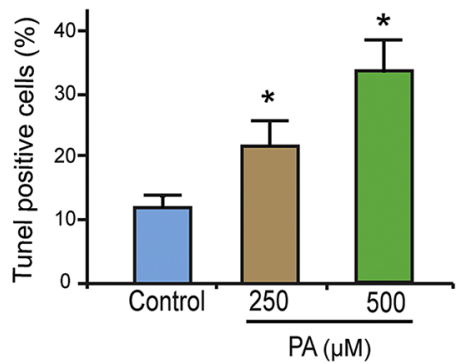


A

Unlabelled

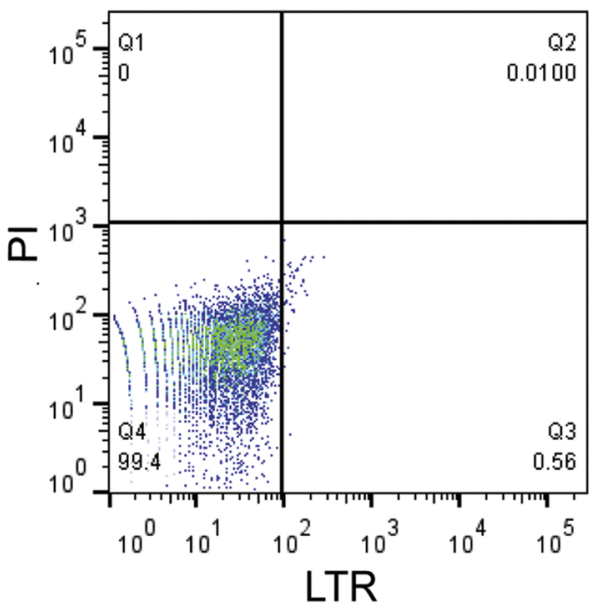

$250 \mu \mathrm{M}$ PA

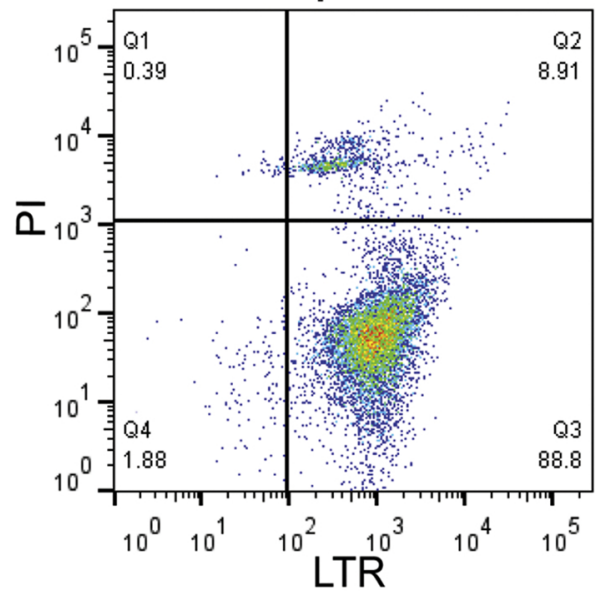

$500 \mu \mathrm{M}$ PA

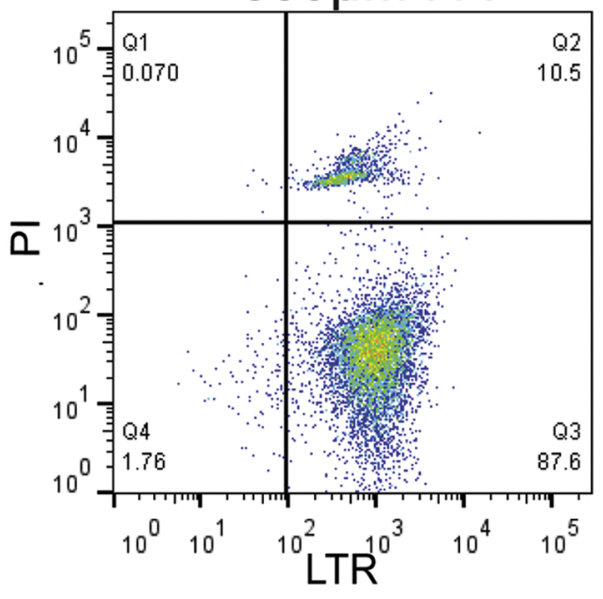

B

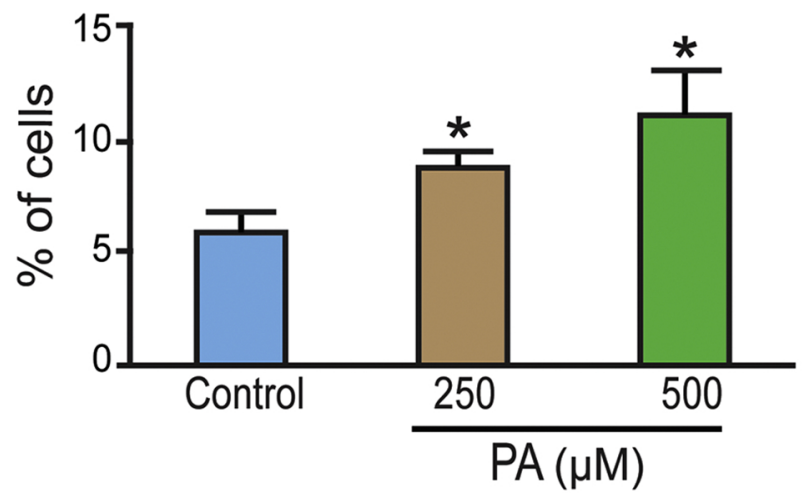

Figure 3 


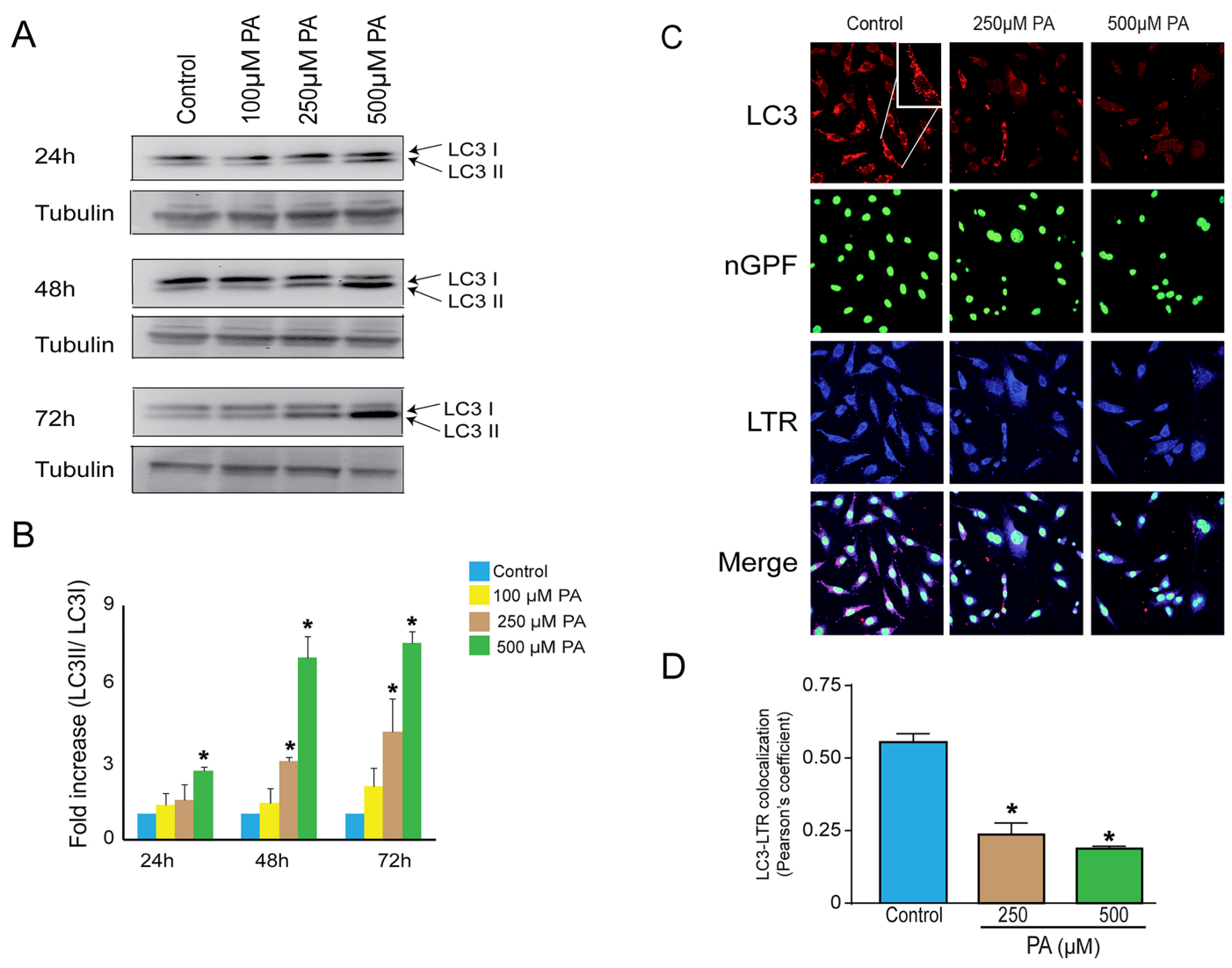

Figure 4 